\title{
Unit of Embryo Infective Dose
}

National Cancer Institute

\section{Source}

National Cancer Institute. Unit of Embryo Infective Dose. NCI Thesaurus. Code C70476.

A unit for measuring infectious activity of a pathogen or biological product such as a

vaccine defined via inoculation in susceptible embryos (e.g. chicken embryos). 